\title{
IMPROVED ERROR BOUNDS FOR BERTRAM'S METHOD
}

\author{
BY BJøRN SUNDT
}

The Wyatt Company, Oslo

\begin{abstract}
In an earlier note the present author deduced bounds for the approximation error of stop loss premiums when the aggregate claims distribution is calculated by a method introduced by Bertram. From the error bounds of the stop loss premiums we deduced bounds for the approximation error of the cumulative distribution and the discrete density of the aggregate claims. In the present note we shall improve the bounds for the cumulative distribution and the discrete density.
\end{abstract}

\section{KEYWORDS}

Aggregate claims distributions; approximations; error bounds.

Let $X$ be the aggregate claims occurred in an insurance portfolio within a given period and $G$ its cumulative distribution. We assume that $X$ is integer-valued and non-negative with finite mean. Let $g$ denote the discrete density and $\bar{G}$ the stop loss transform of $G$, that is,

$$
\begin{array}{ll}
g(x)=\operatorname{Pr}(X=x)=G(x)-G(x-1) & (x=0,1,2, \ldots) \\
\bar{G}(t)=E \max (X-t, 0)=E X-\Sigma_{x=0}^{t-1}(1-G(x)) ; & (t=0,1,2, \ldots)
\end{array}
$$

the latter quantity is the pure premium for an unlimited stop loss treaty with priority $t$.

For a positive integer $m$ we introduce

$$
X_{m}=X-m r_{m}(X)
$$

with $r_{m}(x)$ denoting the largest integer less than or equal to $x / m$. Let $G_{m}$ be the cumulative distribution of $X_{m}$ and $g_{m}$ and $\overline{G_{m}}$ respectively its discrete density and stop loss transform. We easily see that

$$
\begin{aligned}
& 0 \leq X_{m} \leq m-1 \\
& g_{m}(x)=\sum_{k=0}^{\infty} g(x+k m) . \quad(x=0,1, \ldots, m-1)
\end{aligned}
$$

BERTRAM (1981) introduced a method for calculation of compound distributions, by which $g$ is approximated by $g_{m}$ on the range $\{0,1, \ldots, m-1\}$. It is therefore of interest to study how well $g_{m}, G_{m}$, and $\overline{G_{m}}$ approximate $g, G$, and $\bar{G}$. 
Let

$$
\begin{aligned}
& S_{m}=E r_{m}(X)=\sum_{k=1}^{\infty}(1-G(k m-1)) \\
& D_{m}=E\left(X-X_{m}\right)=m S_{m} .
\end{aligned}
$$

In SUNDT (1986) we showed the following inequalities:

$$
\begin{array}{ll}
\overline{G_{m}}(t) \leq \bar{G}(t) \leq \overline{G_{m}}(t)+D_{m} & (t=0,1, \ldots, m-1) \\
G_{m}(x)-D_{m} \leq G(x) \leq G_{m}(x) & (x=0,1, \ldots, m-1) \\
\left|g(x)-g_{m}(x)\right| \leq D_{m} . & (x=0,1, \ldots, m-1)
\end{array}
$$

Formula (3) is a trivial consequence of Lemma 10.1 in SUNDT (1991).

As $E X<\infty$ and $0 \leq m r_{m}(x) \leq x$ for all $x$ and $m$ with $m r_{m}(x)=0$ for $m>x$, we see by bounded convergence that $\lim _{m \uparrow \infty} D_{m}=0$. Thus $\overline{G_{m}}, G_{m}$, and $g_{m}$ converge uniformly towards respectively $\bar{G}, G$, and $g$ when $m$ goes to infinity. We see that if $G(m-1)=1$, then $D_{m}=0$. In that case $g_{m}=g$.

From (1) we see that the stop loss transform $\bar{G}$ satisfies the recursion

$$
\bar{G}(t)=\bar{G}(t-1)+G(t-1)-1 \quad(t=1,2, \ldots)
$$

with initial value $\bar{G}(0)=E X$. Analogously we have

$$
\overline{G_{m}}(t)=\overline{G_{m}}(t-1)+G_{m}(t-1)-1 \quad(t=1,2, \ldots)
$$

with initial value $\overline{G_{m}}(0)=E X_{m}$. It is interesting to note that by applying $E X$ instead of $E X_{m}$ as initial value, we obtain the upper bound in (3) instead of the lower bound. In particular we see that $\bar{G}(t)$ is equal to the upper bound for $t=0$, and thus we believe that $\bar{G}(t)$ is closer to the upper bound than to the lower bound for low values of $t$.

We shall now show that we can replace $D_{m}$ with $S_{m}$ in (4) and (5). In practice $m$ will be a relatively large number, and thus this replacement implies a considerable improvement of the inequalities.

For $x=0,1, \ldots, m-1$ we have

$$
\begin{aligned}
G_{m}(x) & =G(x)+\sum_{k=1}^{\infty}(G(x+k m)-G(k m-1)) \leq \\
& \left.\leq G(x)+\sum_{k=1}^{\infty}(G((k+1) m)-1)-G(k m-1)\right)=G(x)+1-G(m-1)
\end{aligned}
$$

On the other hand, we have $G(x) \leq G_{m}(x)$, and thus

$$
G_{m}(x)-(1-G(m-1)) \leq G(x) \leq G_{m}(x) .
$$

Unfortunately $G(m-1)$ would normally be unknown, and thus we cannot immediately apply the lower bound in (6). However, as $1-G(m-1) \leq S_{m}$, we can replace $1-G(m-1)$ with $S_{m}$ in the lower bound in (6), and thus we obtain

$$
G_{m}(x)-S_{m} \leq G(x) \leq G_{m}(x) .
$$

As

$$
G(x)-\left(G_{m}(x)-S_{m}\right)=\sum_{k=1}^{\infty}(1-G(x+k m))
$$


is decreasing in $x, G(x)$ is closest to the lower bound in (7) for high values of $x$. For low values of $x$, the lower bound might be less than zero, and then it will of course be of no practical interest.

We obviously have $g(x) \leq g_{m}(x)$. On the other hand, by (7)

$$
g(x)=G(x)-G(x-1) \geq G_{m}(x)-S_{m}-G_{m}(x-1)=g_{m}(x)-S_{m},
$$

and we therefore obtain

$$
g_{m}(x)-S_{m} \leq g(x) \leq g_{m}(x) .
$$

In practice $G$ is usually a compound distribution. In that case $E X$ can be calculated as the product of the mean of the counting distribution and the mean of the severity distribution. Unfortunately we will normally need the values of $G_{m}$ to calculate $E X_{m}$ (and thus $D_{m}$ and $S_{m}$ ), and therefore we cannot beforehand determine an $m$ that will give a desired accuracy. What we could do, is to first calculate a rough approximation or upper bound to $S_{m}$ to obtain an idea of how large we should choose $m$. When $g_{m}$ has been found, we calculate the correct value of $S_{m}$.

Let us look at the special case when the tail of $G$ is exponentially bounded, that is, there exist positive constants $C$ and $\kappa$ such that

$$
1-G(x) \leq C e^{-\kappa x}
$$

for all non-negative integers $x$. By applying this inequality to the sum in (2) we obtain

$$
S_{m} \leq \frac{C e^{\kappa}}{e^{\kappa m}-1} \quad D_{m} \leq \frac{m C e^{\kappa}}{e^{\kappa m}-1} .
$$

We see that these bounds approach zero when $m$ approaches infinity.

WiLLmot (1993) deduces an exponential bound for the tail of $G$ for the case when $G$ is a compound geometric distribution.

\section{REFERENCES}

Bertram, J. (1981) Numerische Berechnung von Gesamtschadenverteilungen. Blätter der deutschen Gesellschaft für Versicherungsmathematik XV, 175-194.

SUNDT, B. (1986) Discussion on W. Hürlimann: Error bounds for stop-loss premiums calculated with the Fast Fourier Transform. Scandinavian Actuarial Journal, 114-116.

SuNDT, B. (1991) An introduction to non-life insurance mathematics. (2. ed.) Verlag Versicherungswirtschaft e.V., Karlsruhe.

Willmot, G. E. (1993) On the tails of compound geometric distributions. Research Report 93-02. Institute of Insurance and Pension Research, University of Waterloo.

\section{BJøRn SUNDT}

The Wyatt Company A.S, P.O. Box 1508 Vika, $N-0117$ Oslo, Norway. 